\title{
Breast augmentation for transfeminine patients: methods, complications, and outcomes
}

\author{
Jenna C. Bekeny, Elizabeth G. Zolper, Kenneth L. Fan, Gabriel Del Corral \\ Department of Plastic and Reconstructive Surgery, MedStar Georgetown University Hospital, Washington, DC, USA \\ Contributions: (I) Conception and design: All authors; (II) Administrative support: All authors; (III) Provision of study materials or patients: All \\ authors; (IV) Collection and assembly of data: None; (V) Data analysis and interpretation: None; (VI) Manuscript writing: All authors; (VII) Final \\ approval of manuscript: All authors. \\ Correspondence to: Gabriel Del Corral, MD. MedStar Franklin Square Medical Center, 9000 Franklin Square Dr, Baltimore, MD 21237, USA. \\ Email: drgabrieldelcorral@hotmail.com.
}

\begin{abstract}
Gender-affirming procedures are critical steps in helping transgender patients reach identity actualization and maximal quality of life. Although there are many techniques for gender-affirming care, surgical breast augmentation, or "top surgery," is often cited as the most important—and sometimes onlyprocedure sought by transfeminine patients. Unfortunately, years of individual and systemic prejudice placed barriers between transgender patients and the healthcare providers needed to affirm gender identity. Policy has recently begun to change as research proving the safety, need, and outcomes of breast augmentation in transfeminine patients dismantles long-established systemic inequalities. With this change, more patients are seeking knowledgeable and respectful providers who can address their unique gender-affirming needs. Overall, breast augmentation in transfeminine patients is technically similar to procedures performed in cisgender peers, but with significant considerations. The most common method of augmentation relies on breast implants, since removable prostheses, exogenous hormones, and fat grafting alone often produce unsatisfactory results. Special attention needs to be directed towards anatomic differences in transgender versus cisgender patients in order to achieve optimal size and position of the breast and nippleareolar complex. Complications for transfeminine patients undergoing breast augmentation are rare, and complication rates are equivalent with cisgender peers who pursue similar procedures. Short- and longterm benefits to quality of life have been well-documented. The aim of this review is to give providers the technical knowledge concerning breast augmentation options, pre-surgical evaluation, post-surgical care, and special considerations in transfeminine patients so that provider and patient can have a successful, respectful partnership in reaching gender-affirming goals.
\end{abstract}

Keywords: Transgender; gender dysphoria; hormonal therapy; breast augmentation; chest surgery

Submitted Jan 17, 2020. Accepted for publication Feb 28, 2020.

doi: $10.21037 /$ gs.2020.03.18

View this article at: http://dx.doi.org/10.21037/gs.2020.03.18

\section{Introduction}

In order for transgender patients to receive respectful care, providers must be cognizant of terminology, epidemiology, and the history of transgender healthcare. Gender dysphoria is defined as discrepancy between assigned sex at birth and gender identity that leads to discomfort or distress (1). However, not all individuals who identify as transgender experience gender dysphoria. When this sense persists, some individuals pursue interventions to align their perceived sex with their gender identity and alleviate gender dysphoria.

Although obtaining a true estimate of the transgender population is challenging, transgender persons are estimated to represent $0.4 \%$ to $1.3 \%$ of people worldwide $(2,3)$. There is an estimated slightly higher representation 
of transfeminine persons with 6.8 per 100,000 individuals when compared to transmasculine persons with 2.6 per 100,000 individuals (4). Of note, studies concerning the incidence and prevalence of transgender persons are challenging to perform. These studies tend to specifically capture metrics on transgender persons experiencing gender dysphoria who present to gender centers seeking gender-affirming care in economically developed nations (1). Therefore, patients in disadvantaged socio-economic groups who do not or cannot seek care are not represented. Accurate data collection is further complicated by varying cultural influences and fluctuating expressions of gender identity which may lead to subclinical presentations of gender dysphoria (1). While current research provides a foundation, it most likely underestimates the size of the transgender population.

Despite these challenges, data indicates that the majority of transgender patients ultimately pursue some form of gender-affirming medication or procedure. A retrospective review of patients in an urban community health center by Beckwith et al. noted that almost every transgender patient took exogenous hormones and a third of transgender patients underwent gender-affirming surgery (GAS) (5). It is estimated that as many as $50-70 \%$ of transgender patients seek "top surgery"; thus, the surgical need of this patient population bears epidemiologic significance (6-8).

Gender-affirming care for transgender patients has had a regrettably turbulent course that has only recently begun to correct. Dr. Harry Benjamin wrote in 1941 about his first experience treating a patient with gender dysphoria. $\mathrm{He}$ identified the distinct experience of an adolescent, who felt discordance with assigned gender and noted psychosocial improvement with intake of exogenous hormones (2). Awareness of gender dysphoria and the unique healthcare needs of transgender persons increased with Christine Jorgenson, another of Dr. Benjamin's patients, who was the first American known to have undergone GAS (2). Although Dr. Benjamin laid impressive groundwork for the respectful care of transgender patients, progress came to a halt in 1981. The United States Department of Medicare excluded GAS from covered expenses, blocking a large portion of patients from accessing care (2). In 2014, a complaint by a Medicare beneficiary led to review and repeal of this exclusion, as new studies clearly demonstrated the safety, efficacy, and non-experimental nature of GAS $(2,9)$. Attention to GAS continues to grow as more patients seek care, techniques improve, and research proves the safety, benefit, and cost-effectiveness of these procedures (10).
In this context, gender-affirming care is a critical area for continuing education to improve access to quality care for this vulnerable population. Unfortunately, transgender patients face contrasting rates of stigma, sexual abuse, physical assault, and lethal violence when compared to cisgendered peers (11). In healthcare, transgender patients report higher rates of ill-informed, unsupportive and even hostile caregivers (11). This distrust is promulgated by systemic barriers to care put in place by the government and health insurance groups. By gaining insight into the specialized care of transgender patients, providers will start to bridge the gap and provide equitable service to all patients.

\section{Preoperative planning}

Although preoperative planning is a critical step for any procedure, this phase is especially important when working with patients from the transgender community. Being aware of the context in which transgender patients live, it is essential that providers establish a strong, equitable relationship at the first patient visit. The patient's gender identity must be affirmed which includes physicians inquiring about the patient's preferred name and pronouns (12). The patient's responses may not align with any particular gender or identity category, so it is important to document the patient's preferences. Patients may refer to breast augmentation as "top surgery," and it is also important to acknowledge and document a patient's preferred way of speaking about their procedure.

The World Professional Association for Transgender Health (WPATH) offers guidelines for surgical and nonsurgical gender-affirming care for transfeminine and transmasculine patients. The current, seventh edition outlines both mandatory and suggested components specific to transfeminine top surgery. WPATH requires that welldocumented, persistent gender dysphoria is present in a patient before proceeding with surgical intervention. The patient must be at an age for consent, and the patient must have capacity to give informed consent. All significant medical and mental health concerns must be addressed and managed before surgery.

Involvement of a multidisciplinary team early in the process is required and beneficial for transgender patients. The patient's team should involve physicians with thorough knowledge of transgender health needs and may include members from the disciplines of plastic surgery, endocrinology, primary care, and psychiatry. 
Cisgender patients seeking breast augmentation do not need psychiatric consultation, but one referral from a qualified mental health professional is mandated in order for a transgender patient to pursue breast augmentation (1). While not required, transgender patients are recommended to take exogenous hormones for at least 1 year before undergoing surgical breast augmentation; coordination with an endocrinologist or primary care doctor experienced with transgender patient care is recommended $(1,8)$. The addition of exogenous hormones, can lead to an increase in parenchymal breast tissue, leading to a better cosmetic outcome after breast augmentation.

The pre-operative appointments offer an important opportunity to outline patient goals for breast augmentation. Several studies highlight the incongruity between a provider's definition of an ideal breast augmentation result and a patient's ideal (13). These ideals are shaped by surgeon and patient age, practice type, and country of residence (13). Each patient's ideal outcome should be thoroughly discussed pre-operatively, and providers should discuss limitations to current surgical techniques so that patients have appropriate expectations.

The patient history should include a detailed intake of steps the patient has taken in the transition process. This includes use of exogenous hormones and any prior surgical procedures. As stated before, exogenous hormones should be taken for 1 to 2 years in order to maximize growth of native tissue in preparation for surgical augmentation (8). The most common type of exogenous hormone involves $17-\beta$ estradiol, which is often taken orally in doses of 2 to $6 \mathrm{mg}$ per day, but anti-androgens and other formulations are also commonly used (14). Exogenous estrogens may be stopped 2-4 weeks preceding surgery to reduce risk of thromboembolism (15). However, this remains controversial as stopping exogenous hormones may have deleterious mental health effects on patients and the incidence of thromboembolic events is low (15). In our center, we do not stop exogenous hormones preoperatively for such procedures. We have not found an increase in thromboembolic events. If the decision is made to withhold exogenous hormones, patients must be counseled preemptively to help identify and manage potential sideeffects. Providers must inquire about history of self-obtained exogenous hormones and past "silicone pumping" (11). Unfortunately, the distrust and fear of the medical community coupled with an inability to pay for costly medical procedures leads some transgender patients to pursue treatment from untrained and unmonitored sources (11). If a history of self-obtained substances is disclosed, providers should take this into account when formulating a surgical plan (16). In our practice we see a significant number of patients who undergo illicit silicone injections. Although patients must be warned about the possibility of increased complications, we have not found an increase in any early complications and this should not be considered a contraindication to breast augmentation. Family history must be obtained with a focus on breast cancer incidence in relatives and any known genetic predisposition to breast cancer. Family history of hypercoagulable disorders also informs the decision to withhold estrogens preoperatively.

For both transgender and cisgender patients, it is important to remind patients that surgery devascularizes tissue, so vasoconstrictive agents such as nicotine, cocaine, and methamphetamines must be stopped before undergoing a procedure (15). Smoking cessation is also critical to maximize wound healing capacity in patients (8). Diabetic control is important for cardiovascular and infectious outcomes, so HbA1c should be below $7.0 \%$ before proceeding (15). Any other significant medical or mental health concern must be addressed before pursuing surgical augmentation (1).

\section{Nonsurgical techniques}

Nonsurgical approaches to breast augmentation for transfeminine patients are limited and often have minimal utility. "Prostheses", or removable inserts placed in a brassiere, may be used by transfeminine patients as an initial technique for aligning appearance with identity (15). This approach often does not adequately treat gender dysphoria, and patients usually turn towards more permanent options (15).

Exogenous hormones lead to feminizing changes in breast tissue. The use of exogenous hormones in transfeminine patients does not lead to the same degree of breast nipple areolar complex (NAC) development as genetic females (17). Maximum breast development occurs within the first 3 to 6 months then plateaus between 18 and 36 months $(7,17)$. A prospective multicenter cohort study identified a mean increase in breast-chest difference of $7.9 \mathrm{~cm}(\mathrm{SD} 3.1)$ after a year of exogenous hormone intake with the maximal growth rate occurring in the first 6 months of hormonal therapy (7). This degree of growth corresponds to less than an AAA cup size, with few patients reaching Tanner stage $V(7,17)$. Insufficient breast 


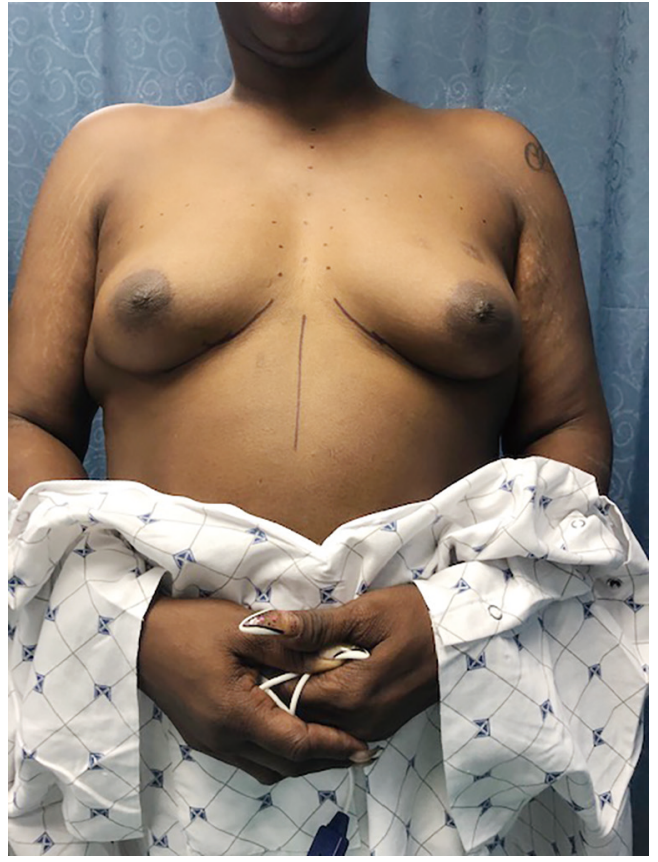

Figure 1 Pre-operative image of a transfeminine patient undergoing implant-based breast augmentation. The breast development seen in this patient is related to exogenous hormone use.

development is cited as the underlying reason to why as many as $50-70 \%$ of transgender patients pursue surgical augmentation in addition to exogenous hormones (6-8).

Even though exogenous hormones are often inadequate as the sole method of breast augmentation, they are a crucial step in the path to surgical augmentation. Patients should take at least 1 year of hormonal therapy before proceeding with definitive surgical procedures $(8,18)$. Hormonal supplementation allows for maximal growth of autologous tissue and allows for skin expansion (18). Since a restrictive skin envelope is an often-cited challenge of transfeminine breast augmentation, it is critical to maximize skin growth preoperatively. See Figure 1 for an example of breast growth secondary to exogenous hormones.

\section{Surgical techniques}

The most common method for transfeminine breast augmentation is placement of implants. Overall, the process is similar between the transgender and cisgender populations with additional considerations for transfeminine patients.

There are discrepancies between genetic male and genetic female anatomy that must be discussed preoperatively and accounted for intraoperatively in order to produce a satisfactory result. The genetic male anatomy is typified by small, ovoid, lateralized NAC, hypertrophied pectoralis major muscle, wide sternum, and shortened distance between the nipple and inframammary fold (IMF) $(3,13)$. Breast augmentation will stretch the overlying skin, increasing NAC size to a degree; however, this often does not result in a fully feminized appearance of the NAC (13). Due to the lateralized NAC, a precarious balance exists between achieving maximal cleavage and developing a centralized NAC position (13). It is important to counsel patients preoperatively about the possibility of increased nipple lateralization after breast augmentation. Most patients would like to achieve cleavage without clothing; it is imperative to counsel patients that this may not be possible due to the base diameter of the breast. Placing implants that defy anatomical restraints may lead to increased secondary complications and soft tissue abnormalities. Periareolar mastopexy is an option to achieve a more medial or "feminine" nipple location. It is important for patients to understand that this can jeopardize nipple sensation; moreover, this sort of procedure is challenging to obtain insurance authorization. Surgeons should inquire about which aspects are important for achieving a patient's desire result.

A hypertrophied pectoralis major muscle affects the aesthetic results of certain planes of implantation. The subpectoral plane is often used to avoid rippling produced by implantation in the prepectoral plane (13). However, larger pectoralis major muscles will produce a wider breast base that may be displeasing to some patients. In our practice, with very hypertrophic pectoralis muscle, we have found that over time implants tend to lateralize. In these patients a subglandular augmentation may be preferred. Years of exposure to androgenic stimulation yields patients with broadened shoulders and widened sternums (13). Without compensation for these anatomic differences, patients may end up with lateralized augmented breasts with less than ideal cleavage. One method of compensation involves elevation of select medial pectoralis major fibers in order to avoid lateralization. Caution must be used if this method is undertaken, since over-elevation can lead to medial implant migration which is challenging to correct (13). Overall, shared-decision making can uncover which aspects of breast and NAC appearance are of most importance, so that surgeons can prioritize these considerations and address limitations preoperatively. 


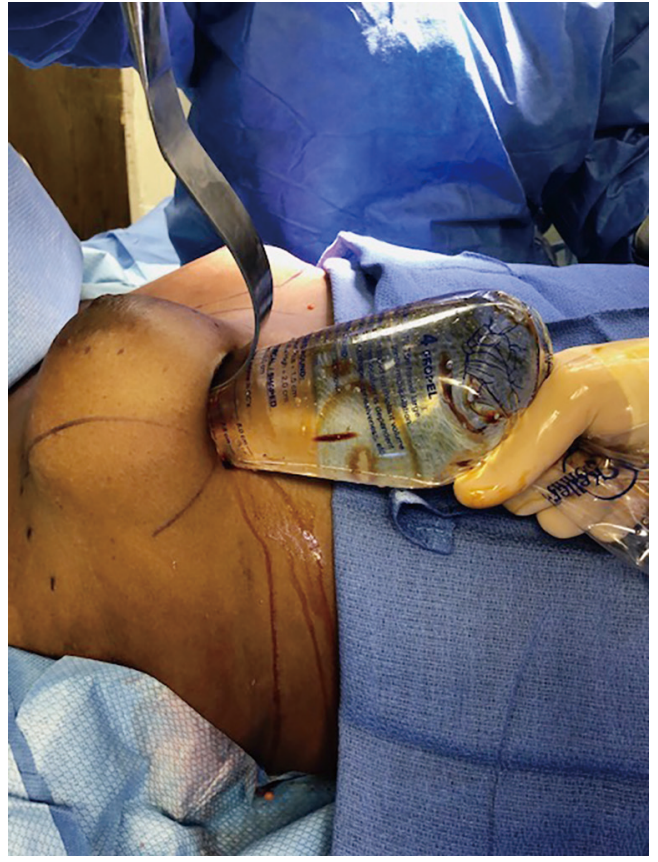

Figure 2 Insertion of breast implant through inframammary incision. Multiple approaches to implant-based breast reconstruction are available, and a thorough understanding of risks and benefits are needed by provider and patient.

Methodologies specific to selecting implant characteristics in the transfeminine population have not been developed or validated. However, surgeons report success using methodologies validated in the cisgendered population with the transgender population $(19,20)$. The TEPID and the simplified High Five decision support process outline important considerations for implant-based breast augmentation (20). These considerations include soft tissue coverage, implant volume, type, size, and dimensions, incision location, and IMF location (20). The landmark publication by Kanhai et al. highlights desired implant size disparities between transgender and cisgender patients. Over the course of nearly two decades, the average implant size increased by more than 1.5 times for transgender patients (21). This desired outcome needs to be balanced with the paucity of soft tissue and skin coverage described earlier.

Of note, there are mastectomy guidelines specific to the transgender population which have been proposed based on breast size and envelope of the transmasculine patient $(22,23)$. Different mastectomy approaches were found to have disparate rates of revision due to aesthetic concerns and surgical complications which led to these guidelines $(24,25)$. As more data is compiled on breast augmentation in the transfeminine population, development of transgender specific evidence-based guidelines will be critical to optimizing outcomes, complications, and patient satisfaction in this population.

Incision location can be axillary, inframammary, or periareolar (18). For transfeminine patients, periareolar incisions are more difficult as the NAC is small relative to cisgender patients (18). If an inframammary incision is used, it should be positioned lower than the native IMF preoperatively as placement of implants expands the inferior pole and creates a new fold (18). See Figure 2 for an example of inframammary placement. The Akademikliniken method (AK or Q2), proposed by Per Hedén, addresses this issue through preoperative assessment of two distancesthe distance between the NAC and the IMF and vertical distance between the implant and the NAC $(26,27)$. The ICE method, proposed by Mallucci and Branford, is a simplified, prospectively validated approach to inframammary incisionbased implants. The ICE method stands for a calculation with three characteristics-implant dimensions (I), breast capacity $(\mathrm{C})$, and excess tissue (E). The calculation is $\mathrm{I}-\mathrm{C}=\mathrm{E}$, where $\mathrm{I}$ is half of the implant height plus the projection, $\mathrm{C}$ is nippleto-IMF distance on stretch, and $\mathrm{E}$ is the distance by which the IMF must be lowered (28). The method is useful for creating a breast with "ideal characteristics", such as an upper to lower pole ratio of $45 \%$ to $55 \%$ and upward nipple angulation of $20^{\circ}(28)$.

Decisions about implant plane have particular considerations for the transgender population. The prepectoral plane may be preferred in patients with more mature glandular tissue. Patients with Tanner stage IV or $\mathrm{V}$ may present with sufficient soft tissue for prepectoral placement which allows for a relatively less painful operation (18). However, if prepectoral placement is chosen, attention must be paid to the increased distance between the NAC so that overtly lateral breasts are not constructed (21). There is also an increased risk of capsular contracture in patients with prepectoral augmentations. This is important to discuss with patients preoperatively as they may require future procedures, such as capsulectomies. This type of revisional procedure is challenging to achieve insurance coverage, and patients need to be aware of this. Patients with less mature tissue or thinner body habitus may be better candidates for subpectoral implantation. If subpectoral placement is pursued, patients may be at lowerrisk for capsular contracture. However, this procedure 


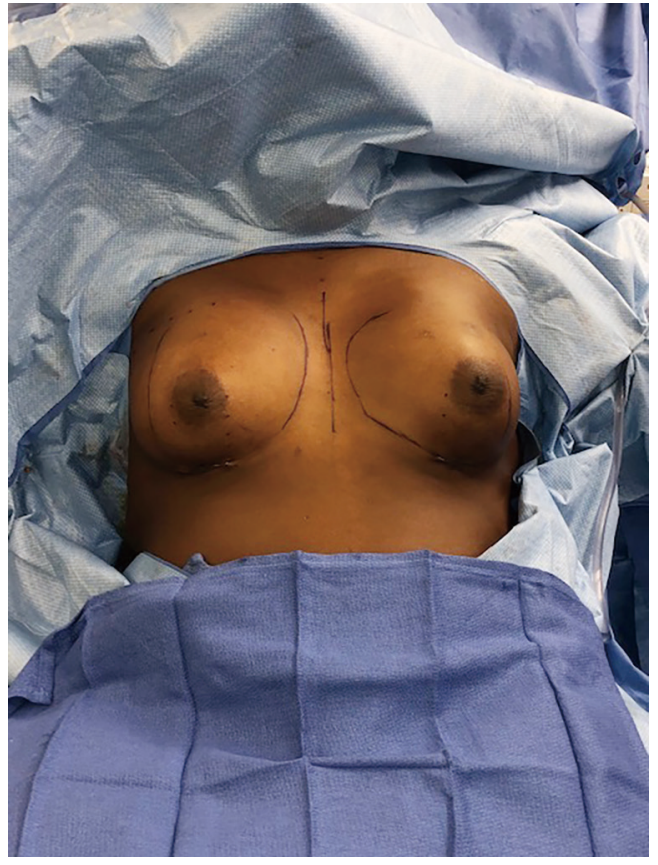

Figure 3 Immediate post-operative image of a transfeminine patient with implant-based breast augmentation. With time, implants will settle.

requires selective elevation of the inferior and medial borders of the pectoralis major to achieve appropriate implant placement (18). Pectoralis major hypertrophy can lead to progressive lateralization of the implant over-time, so this should be monitored for in patients with subpectoral implants (21).

Although rarely used as the only method for breast augmentation in transgender patients, fat grafting can be used as an adjunct to implants (15). Fat grafting is especially useful for developing more natural appearing cleavage and making implants less visible or palpable $(15,18)$. Reported take rates in fat grafting range from $50-60 \%$, so patients should be counseled that additional procedures are often necessary to achieve the desired breast volume $(8,18)$. Common donor sites for fat grafting are the abdomen, thighs, and flanks (8).

Even more infrequently used for breast augmentation in the transgender female population is the use of autologous tissue. A case report by Majdak-Paredes et al. describes a transgender female who noticed asymmetrical breast development after taking a year-long course of exogenous hormones. Examination revealed a hypoplastic left breast and absent left pectoralis major muscle, supporting a diagnosis of Poland syndrome. After reviewing options for breast reconstruction, the patient underwent tissue expansion followed by a transverse rectus abdominis myocutaneous flap without complication. The patient was satisfied with the procedure, but opted to undergo additional procedures to correct a contour defect above the left NAC and to reposition the size of the right NAC (29).

\section{Postoperative care}

Postoperative care of transfeminine patients undergoing breast augmentation is similar to cisgender female patients with a few specific considerations. Transgender patients have historically faced prejudice from the medical community, and the postoperative period presents a potential arena for sensitivity (30). All medical staff members in the postanesthesia care unit need to be trained to respectfully care for transgender patients undergoing breast augmentation (30). Appropriate short-term care involves proper brasserie fitting and implant massage to soften the skin envelop (15). Activity restrictions vary across institutions, but it is generally recommended that patients avoid lifting more than five pounds and high-impact activities for a minimum of 4-6 weeks post-operatively (8). See Figure 3 for postoperative results.

In terms of long-term postoperative care, there are a few considerations for all patients-transgender or cisgenderwho have breast implants. According to the FDA, breast implants are not classified as lifetime devices, so they require monitoring and eventual replacement. For patients with silicone implants, magnetic resonance imaging (MRI) screening for silent rupture should occur 3 years after implantation and every 2 years after the first screening (8). Patients should be made aware of the need to replace implants and timing of planned implant exchange should be discussed.

Cancer screening for transfeminine patients is often concordant with cisgender females. Recommendations suggest breast cancer screening for transfeminine patients who have taken hormones for 5 or more years and are 50 years of age or above (3). Although rare, there is a growing body of knowledge about breast implant-associated anaplastic large cell lymphoma (BIA-ALCL) in transgender patients with textured breast implants (31-33). Presenting signs and symptoms of BIA-ALCL are similar in the transfeminine population as the cisgender population, including overlying pigmentation changes, pruritus, newonset breast mass development, and rash (32). Early 
diagnosis and treatment are critical, so patients with history of textured implants should be notified of the risks and presenting symptoms.

\section{Complications, outcomes, and barriers to care}

Research shows that breast augmentation in the transfeminine population has complication rates similar to those in breast augmentation for the cisgender population (34). Cuccolo et al. reviewed a decade of data from the National Surgical Quality Improvement Program and discovered no significant difference in 30day complication rates for transgender and cisgender breast augmentation patients, even though the transgender cohort was older, more comorbid, and had higher average body mass index. Complication rates for both populations were low, with an all cause complication rate of $1.8 \%$ in the transfeminine population and $1.6 \%$ in the cisgender population (34). Reasons for reoperation were similarly low, and included hematoma and abscess drainage, with rates of $1.1 \%$ and $0.4 \%$, respectively (34). The most common complications specific to breast implantation in both the cisgender and transgender population include symmastia, capsular contracture, reduced breast or nipple sensation, implant leakage, or implant migration (18). Other complications include the common surgical complicationswound dehiscence, seroma, hematoma, and infection. While rare, galactorrhea has been reported, and requires extensive hormonal evaluation if detected (18). Overall, implantbased breast augmentation remains a safe procedure in the transfeminine population.

Surgical options for breast augmentation in the transfeminine population also have positive short- and long-term effects on patient quality of life. An analysis by Weigert et al. showed statistically significant improvements in BREAST-Q scores 4 and 12 months after surgical breast augmentation in a prospective cohort of transfeminine patients. Of note, scores improved in categories of breast satisfaction, psychosocial well-being, and sexual wellbeing at both time points (35). Similar studies also indicate that the transfeminine population benefits from breast augmentation procedures and that these results have lasting effects on patient well-being $(36,37)$.

While rare, there is growing attention paid to the subset of patients undergoing gender-affirming care who later express regret or pursue reversal procedures. A 30 year study identified $0.6 \%$ of transgender females and $0.3 \%$ transgender males who felt regret about gonadal procedures (38). However, regret after surgical procedures of the chest has not been reported in the literature. Factors related to regret are often based in failure to meet expectations, complications, and lack of psychosocial support $(39,40)$. Other factors include older patient age, social instability, heterosexual orientation, dissatisfaction with aesthetics and function, and poor partner or familial support (39).

\section{Conclusions}

In order to provide high-quality care for all patients, healthcare providers need to be familiar with the experience, concerns, and goals of transgender patients. Decades of exclusion from the healthcare system have placed barriers between providers and patients. As historical and systemic prejudices have begun to be undone, more patients are beginning to seek medical and surgical options for gender-affirming care. Breast augmentation is an important and sometimes singular step in affirming a transfeminine individual's identity. Non-surgical options are limited and often yield insufficient patient satisfaction. Surgical options are more common, and often utilize breast implants with decisions on sizing, implant location, and incision type made based on protocols developed for the cisgender population. Research indicates that breast augmentation in the transfeminine population is as safe a procedure as when performed in cisgendered patients, and there are positive short- and long-term effects for patient well-being. However, there are unique anatomic and psychosocial considerations to be aware of when performing augmentation for transfeminine patients in order to achieve maximal satisfaction. With an increasing body of knowledge, physicians will be able to appropriately and respectfully care for transgender patients. In time, providers can work to close the gap in care and provide transgender patients with favorable and equitable treatment outcomes.

\section{Acknowledgments}

Funding: None.

\section{Footnote}

Conflicts of Interest: All authors have completed the ICMJE uniform disclosure form (available at http://dx.doi. org/10.21037/gs.2020.03.18). The authors have no conflicts of interest to declare. 
Ethical Statement: The authors are accountable for all aspects of the work in ensuring that questions related to the accuracy or integrity of any part of the work are appropriately investigated and resolved.

Open Access Statement: This is an Open Access article distributed in accordance with the Creative Commons Attribution-NonCommercial-NoDerivs 4.0 International License (CC BY-NC-ND 4.0), which permits the noncommercial replication and distribution of the article with the strict proviso that no changes or edits are made and the original work is properly cited (including links to both the formal publication through the relevant DOI and the license). See: https://creativecommons.org/licenses/by-nc-nd/4.0/.

\section{References}

1. Coleman E, Bockting W, Botzer M, et al. Standards of Care for the Health of Transsexual, Transgender, and Gender-Nonconforming People. Int J Transgend 2012;13:165-232.

2. Berli JU, Knudson G, Fraser L, et al. What Surgeons Need to Know About Gender Confirmation Surgery When Providing Care for Transgender Individuals: A Review. JAMA Surg 2017;152:394-400.

3. Safa B, Lin WC, Salim AM, et al. Current Concepts in Feminizing Gender Surgery. Plast Reconstr Surg 2019;143:1081e-91e.

4. Arcelus J, Bouman WP, Van Den Noortgate W, et al. Systematic review and meta-analysis of prevalence studies in transsexualism. Eur Psychiatry 2015;30:807-15.

5. Beckwith N, Reisner SL, Zaslow S, et al. Factors Associated with Gender-Affirming Surgery and Age of Hormone Therapy Initiation Among Transgender Adults. Transgend Health 2017;2:156-64.

6. Miller TJ, Wilson SC, Massie JP, et al. Breast augmentation in male-to-female transgender patients: Technical considerations and outcomes. JPRAS Open 2019;21:63-74.

7. de Blok CJM, Klaver M, Wiepjes CM, et al. Breast Development in Transwomen After 1 Year of Cross-Sex Hormone Therapy: Results of a Prospective Multicenter Study. J Clin Endocrinol Metab 2018;103:532-8.

8. Van Boerum MS, Salibian AA, Bluebond-Langner R, et al. Chest and facial surgery for the transgender patient. Transl Androl Urol 2019;8:219-27.

9. Division A, Appeals Board of Health Services. Department of Health and Human Services DEPARTMENTAL APPEALS BOARD Appellate Division. 2014. Available online: https://www.hhs.gov/sites/default/files/static/dab/ decisions/board-decisions/2014/dab2576.pdf

10. Padula WV, Heru S, Campbell JD. Societal Implications of Health Insurance Coverage for Medically Necessary Services in the U.S. Transgender Population: A Cost-Effectiveness Analysis. J Gen Intern Med 2016;31:394-401.

11. Winter S, Diamond M, Green J, et al. Transgender people: health at the margins of society. Lancet 2016;388:390-400.

12. Wesp LM, Deutsch MB. Hormonal and Surgical Treatment Options for Transgender Women and Transfeminine Spectrum Persons. Psychiatr Clin North Am 2017;40:99-111.

13. Morrison SD, Wilson SC, Mosser SW. Breast and Body Contouring for Transgender and Gender Nonconforming Individuals. Clin Plast Surg 2018;45:333-42.

14. Abramowitz J, Tangpricha V. Hormonal Management for Transfeminine Individuals. Clin Plast Surg 2018;45:313-7.

15. Narayan SK, Morrison T, Dugi DD 3rd, et al. Gender Confirmation Surgery for the Endocrinologist. Endocrinol Metab Clin North Am 2019;48:403-20.

16. Leonardi NR, Compoginis JM, Luce EA. Illicit Cosmetic Silicone Injection: A Recent Reiteration of History. Ann Plast Surg 2016;77:485-90.

17. Maycock LB, Kennedy HP. Breast care in the transgender individual. J Midwifery Womens Health 2014;59:74-81.

18. Claes KEY, D’Arpa S, Monstrey SJ. Chest Surgery for Transgender and Gender Nonconforming Individuals. Clin Plast Surg 2018;45:369-80.

19. Hidalgo DA. Breast augmentation: choosing the optimal incision, implant, and pocket plane. Plast Reconstr Surg 2000;105:2202-16; discussion 2217-8.

20. Tebbetts JB, Adams WP. Five critical decisions in breast augmentation using five measurements in 5 minutes: the high five decision support process. Plast Reconstr Surg 2005;116:2005-16.

21. Kanhai RCJ, Hage JJ, Asscheman H, et al. Augmentation mammaplasty in male-to-female transsexuals. Plast Reconstr Surg 1999;104:542-9.

22. Cregten-Escobar P, Bouman MB, Buncamper ME, et al. Subcutaneous mastectomy in female-to-male transsexuals: a retrospective cohort-analysis of 202 patients. J Sex Med 2012;9:3148-53.

23. Monstrey S, Selvaggi G, Ceulemans P, et al. Chest-wall contouring surgery in female-to-male transsexuals: A new algorithm. Plast Reconstr Surg 2008;121:849-59.

24. Bluebond-Langner R, Berli JU, Sabino J, et al. Top 
Surgery in Transgender Men: How Far Can You Push the Envelope? Plast Reconstr Surg 2017;139:873e-82e.

25. van de Grift TC, Elfering L, Bouman MB, et al. Surgical indications and outcomes of mastectomy in transmen: A prospective study of technical and self-reported measures. Plast Reconstr Surg 2017;140:415e-24e.

26. Montemurro P, Agko M, Li AQ, et al. Implementation of an integrated biodimensional method of breast augmentation with anatomic, highly cohesive silicone gel implants: Short-term results with the first 620 consecutive cases. Aesthet Surg J 2017;37:782-92.

27. Hedén P. Invited Discussion on: Tissue-Based Implant Selection and Preoperative Markings with the AK or Q2 Method. Aesthetic Plastic Surgery. Springer New York LLC, 2019.

28. Mallucci P, Branford OA. Design for Natural Breast Augmentation: The ICE Principle. In: Plastic and Reconstructive Surgery. Lippincott Williams and Wilkins, 2016:1728-37.

29. Majdak-Paredes EJ, Shafighi M, Fatah F. Unilateral hypoplastic breast in a male-to-female transsexual with Poland syndrome after gender reassignment-reconstructive considerations. J Plast Reconstr Aesthet Surg 2009;62:398-401.

30. Tollinche LE, Burrows Walters C, Radix A, et al. The perioperative care of the transgender patient. Anesth Analg 2018;127:359-66.

31. de Boer M, van der Sluis WB, de Boer JP, et al. Breast Implant-Associated Anaplastic Large-Cell Lymphoma in a Transgender Woman. Aesthet Surg J 2017;37:NP83-7.

32. Ali N, Sindhu K, Bakst RL. A Rare Case of a Transgender Female With Breast Implant-Associated Anaplastic Large Cell Lymphoma Treated With Radiotherapy and a Review of the Literature. J Investig Med High Impact Case Rep

Cite this article as: Bekeny JC, Zolper EG, Fan KL, Del Corral G. Breast augmentation for transfeminine patients: methods, complications, and outcomes. Gland Surg 2020;9(3):788796. doi: $10.21037 /$ gs.2020.03.18
2019;7:2324709619842192.

33. Patzelt M, Zarubova L, Klener P, et al. Anaplastic LargeCell Lymphoma Associated with Breast Implants: A Case Report of a Transgender Female. Aesthetic Plast Surg 2018;42:451-5.

34. Cuccolo NG, Kang CO, Boskey ER, et al. Epidemiologic Characteristics and Postoperative Complications following Augmentation Mammaplasty: Comparison of Transgender and Cisgender Females. Plast Reconstr Surg Glob Open 2019; 7:e2461.

35. Weigert R, Frison E, Sessiecq Q, et al. Patient satisfaction with breasts and psychosocial, sexual, and physical wellbeing after breast augmentation in male-to-female transsexuals. Plast Reconstr Surg 2013;132:1421-9.

36. Lindqvist EK, Sigurjonsson H, Möllermark C, et al. Quality of life improves early after gender reassignment surgery in transgender women. Eur J Plast Surg 2017;40:223-6.

37. Becker I, Auer M, Barkmann C, et al. A Cross-Sectional Multicenter Study of Multidimensional Body Image in Adolescents and Adults with Gender Dysphoria Before and After Transition-Related Medical Interventions. Arch Sex Behav 2018;47:2335-47.

38. Wiepjes CM, Nota NM, de Blok CJM, et al. The Amsterdam Cohort of Gender Dysphoria Study (19722015): Trends in Prevalence, Treatment, and Regrets. J Sex Med 2018;15:582-90.

39. Djordjevic ML, Bizic MR, Duisin D, et al. Reversal surgery in regretful male-to-female transsexuals after sex reassignment surgery. J Sex Med 2016;13:1000-7.

40. Landén M, Wålinder J, Hambert G, et al. Factors predictive of regret in sex reassignment. Acta Psychiatr Scand 1998;97:284-9. 